Feast: In answer to Dr. Buscombe I think it would be generally admitted that Of stars are probably an example of mild WR activity.

Buscombe: Less luminous, however.

Feast: We don't know the lower limit to WR stars in the Galaxy.

Buscombe: Existing plate material should be surveyed for novae that have recently been overlooked in the Magellanic Clouds.

Ambartsumian: What is the faint limit of your survey of WR stars especially in and around the region of 30 Doradus?

Westerlund: Inside 30 Doradus my identification depends entirely on Thackeray's results as we cannot identify WR stars, because of the emission nebulosity on objective prism plates. Outside, the faintest is about 15th magnitude.

Aller: Magnitudes of planetaries secured with a broad band-pass filter are difficult to interpret in terms of monochromatic fluxes. With regard to $\mathrm{B}$ magnitudes, one notes that the ratio of the green nebula [OIII] lines to $\mathbf{H} \beta$ ranges from considerably less than 1 (NGC 40) to around 20.

Westerlund: I have discussed this point with Baade and Minkowski. They agreed that one must use B magnitudes for statistical purposes.

Eggen: Is it not possible that there is a difference for WR stars inside and outside 30 Doradus?

Westerlund: It is possible but I do not think that the effect is seen on slit spectra of the objects.

\title{
66. SUPERGIANTS IN THE MAGELLANIC CLOUDS AND IN THE GALAXY
}

\author{
Th. WaLraven and J. H. Walraven \\ Mount Stromlo Observatory
}

\section{Introduction}

With the five-colour photometer of the Leiden Southern Station (system $V, B, L, U, W$ ) stars can be classified according to luminosity (Th. Walraven and J. H. Walraven 1960).

The method was used in a search for member stars of the Magellanic Clouds, which are supergiants and can be easily distinguished from the galactic foreground stars, which are dwarfs. In addition, already recognized members from the list of Feast, Thackeray, and Wesselink (1960), and O-B stars from the HDE catalogue were observed. In total about 600 stars were inspected, of which roughly $50 \%$ turned out to be members. The result of this investigation was read in a paper at the IAU Symposium XI, at Berkeley, but it was not published in detail; we decided to complete the work with the investigation of many more bright galactic stars of different spectral type and luminosity. This program included approximately 300 stars.

In this paper we give only some results of a comparison of the Magellanic stars with the galactic supergiants and the detailed results and catalogue of stars will be published in the Bulletin of the Astronomical Institutes of the Netherlands. 


\section{The Colour-Magnitude Relation $m_{V}$ versus $m_{B}-m_{V}$}

The stars belonging to the Clouds occupy a continuous band, which for faint stars $\left(m_{V}=15\right)$ fits the main sequence type B0 or B1. For brighter stars this band bends off towards redder colour in a way similar to that shown by the galactic cluster $h$ and chi Persei. The brightest stars form a horizontal array, covering a wide range of colour. These stars are mostly between 10th and 11th magnitude. Below the horizontal sequence, i.e. between 12th and 15th, practically every yellow star is a foreground star. In the diagram the bluer members of the Clouds are separated from the yellow foreground stars by a gap, for increasing colour index the members of the Clouds are rapidly decreasing in number just before the yellow galactic foreground stars appear in large numbers. For red stars (about type K0) we cannot distinguish members from the foreground stars, because the luminosity effects become small and the ultraviolet regions $U$ and $W$ are too faint for accurate observations. For these reasons red stars were not studied. However, our results indicate at least that yellow giants, between $m_{V}=12$ and $m_{V}=15$, are exceedingly rare in the Magellanic Clouds.

The vertical band of blue and white members of the Clouds is about $0{ }^{\mathrm{m}} 4$ wide in colour $m_{B}-m_{V}$. In the next section we show that this is not caused by interstellar reddening, but by variations in intrinsic colours of the stars.

\section{The Colour-Colour Relations}

In this section we show only the relation $m_{B}-m_{V}$ versus $m_{U}-m_{B}$ (see Fig. 1). The stars were grouped in intervals of one magnitude and mean lines of constant magnitude drawn for each group.

Line $a$ represents the mean of the stars with $10<m_{V}<11$, line $b$ is for $11<m_{V}<12$, and so on. The dashed portions of the lines are uncertain owing to limited numbers of stars or large scatter.

The foreground stars are detected by their position on the main sequence line instead of on the lines $a$ to $e$. Where these lines cross the main sequence, other colour diagrams are used to decide their membership. For the red stars, where the lines cross again the main sequence, the other diagrams are of no use.

Before drawing the mean lines of constant magnitude, a large number of stars were excluded, all showing positive excesses in $m_{B}-m_{V}$. These are:

(1) Stars of "P Cygni" type or with emission,

(2) stars appreciably reddened by dust,

(3) composite stars,

(4) peculiar stars.

Most of the rejected stars fall in group 1. The different cases can be distinguished from the four colour excesses, if these are larger than the observational errors. It is curious that so many different causes all lead to an apparent reddening of the stars in $m_{B}-m_{V}$ and none otherwise.

The lines in Figure 1 are corrected for galactic interstellar reddening by an amount of 0 m05 in $m_{B}-m_{V}$, which was determined from foreground stars. 
The deviations between observed magnitude and the magnitude read in Figure 1 at the observed colours have a mean value of 0.5 or, vice versa, the deviations in colour $m_{B}-m_{V}$ have a mean value of 0 m028. This scatter could be produced by several causes, which are:

(1) Variations in interstellar reddening (in the Clouds),

(2) cosmic scatter in the relation between luminosity and colours,

(3) weak emission or "P Cygni" effects,

(4) variations in distance,

(5) observational errors.

Among the excluded stars discussed above, emission features occur more often than strong reddening by dust and its effect on the colours can be equally large. In the case of the small deviations where the inaccuracy of the observations

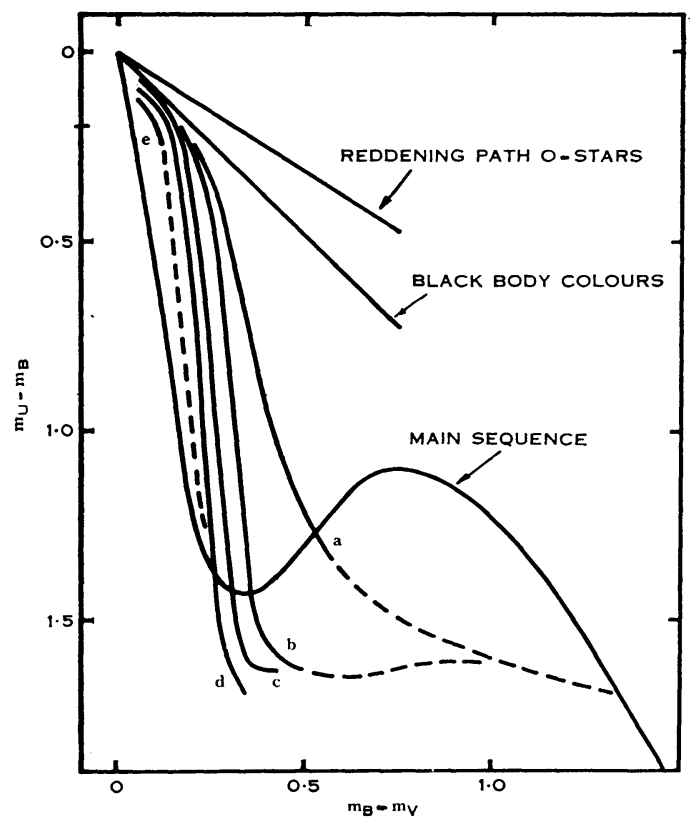

Fig. 1.-The colour-colour diagram $m_{B}-m_{V}$ versus $m_{U}-m_{B}$. Magellanic stars: $a, 10<m<11$; $b, \quad 11<m<12 ;$ c, $12<m<13 ; d, 13<m<14$; $e, 14<m<15$. Main sequence for galactic dwarfs.

especially in $W$ does not permit a distinction between the various effects, it is difficult to decide which is the most important effect or whether a systematic reddening by dust is still present in the mean lines of constant magnitude. Since we estimate that this can not be more than $0 \mathrm{~m} .02$ we may consider Figure 1 as the best approach to the intrinsic colours of normal stars in the Clouds.

The most remarkable feature besides the gradual reddening with the increasing luminosity is the sudden decrease in maximum Balmer jump from the line $b$ to the line $a$. According to theoretical models (e.g. de Jager and Neven 1957) the maximum 
Balmer jump steadily increases from the main sequence stars towards the giants. This is indeed observed since the maximum Balmer jump for groups $b, c$, and $d$ is appreciably stronger than for the main sequence. However, for supergiants the maximum Balmer jump no longer increases and for the most luminous supergiants even decreases rather suddenly. At the same time the scatter in the Balmer jump increases (Fig. 3). The galactic supergiants show the same phenomenon. Although the interstellar reddening for these stars varies strongly, one can still distinguish the deep from the less deep Balmer jumps and it was found that the observed galactic supergiants of type F or G, class Ia (e.g. $\delta \mathrm{CMa}, \mathrm{HD} 90772, \mathrm{HD} 97534$, and b Vel) belong to the same group $a$, whereas stars of class Iab or Ib (e.g. $\alpha$ Lep, HD 80404, HD 75276) have much larger Balmer jumps and fall at the lower tip of lines $b, c$ or $d$.

The relation between colour index $m_{B}-m_{V}$ and luminosity can easily be interpreted. For the hotter supergiants the atmospheric opacity is almost entirely due to electron scattering. Since the hydrogen is mainly ionized the Balmer jump is small and proportional to the percentage of neutral hydrogen atoms. The decrease of pressure connected with the increase in luminosity has to be compensated by decrease of temperature in order to maintain a constant small value of the Balmer jump.

A rough estimate, involving use of the mass-luminosity relation, the formula for equilibrium of ionization and excitation, and the law of black body radiation, shows that the expected increase of colour index with increasing luminosity is indeed of the same order as the observed effect.

For larger values of the Balmer jump this simple approximation is no longer valid. Although Figure 1 suggests that the separation between the lines for constant magnitude is continued for cooler stars, it is at present impossible to give satisfactory interpretation of the wide separation between the lines $a$ and $b$ for the cooler stars.

\section{The Colour-Spectrum Relation}

For many of the brighter stars of our list, spectral types have been determined at the Radcliffe Observatory. The stars of type A0 are so numerous that an increase in colour index $m_{B}-m_{V}$ for increasing luminosity can be definitely established. At the same time the colour $m_{U}-m_{B}$ becomes bluer. The less luminous supergiants of type A0 have about the same colour $m_{B}-m_{V}$ as stars of type A0 V. Faint supergiants of type $F$ are bluer than the main sequence $F$ stars, but bright supergiants on the average are redder.

Very notable is the enormous difference in colour between $G$ type dwarfs and supergiants. Whilst G5 V stars have a colour index $m_{B}-m_{V}=1 \stackrel{\mathrm{m}}{0} 00$, the Magellanic supergiant HDE 268757, type G5 Ia, has $m_{B}-m_{V}=2$ m06, i.e. more than a magnitude redder.

In Figure 2 these effects are shown. The upper part gives the colour excess, relative to class $\mathrm{V}$, for bright supergiants, the middle part for fainter stars. The bottom part of the figure shows Johnson's relation for galactic supergiants (1958). In the $V, B, L, U, W$ system the colour $m_{B}-m_{V}$ becomes for later types gradually 
redder than the colour $B-V$ of Johnson's photometer system and the difference increases to 0.2 for $M$ type stars. Therefore the similarity between Johnson's curve and those for the Magellanic stars in fact is even closer than is shown in Figure 2. So also with respect to intrinsic colour the Magellanic supergiants and the galactic supergiants behave in a similar way.

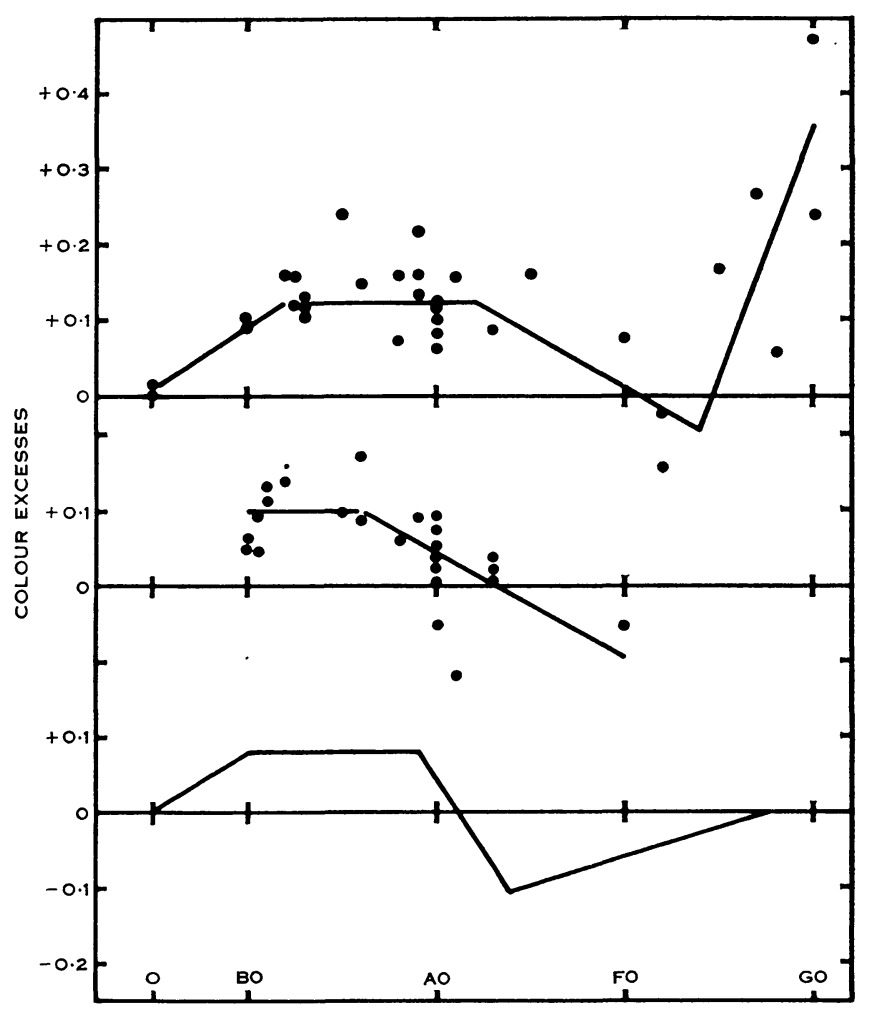

Fig. 2.-Colour-excesses of supergiants relative to class V, as a function of spectral type.

\section{The Reduced Colour-Colour Relation}

Up to now no direct comparison could be made between the Magellanic Cloud and galactic supergiants, owing to the strong unknown reddening of the latter. However, we can use the reduced colours $[B-U],[B-L]$, and $[U-W]$, which are defined in the same way as the $\mathrm{Q}$ of the $U, B, V$ system. In the diagram $[B-L]-$ [B-U] the groups $a, b$, and $c$ give the same line for all supergiants between B3 and A0, i.e. no luminosity effect is seen, although the scatter of the points is very low. For types later than A0 the line $a$ diverges suddenly from the lines $b$ and $c$ (see Fig. 3). As can be seen in Figure 3 the galactic supergiants again follow the same pattern. In Figure 3 the above-mentioned large scatter in the Balmer jump for the later type stars is most clearly demonstrated. 
In the relation $[U-W]-[B-U]$ a very pronounced luminosity effect is present, as is shown in Figure 4, where the long vertical lines represent the mean for groups $a, b$, and $c$. In this diagram the lines are not continued for types later than F0.

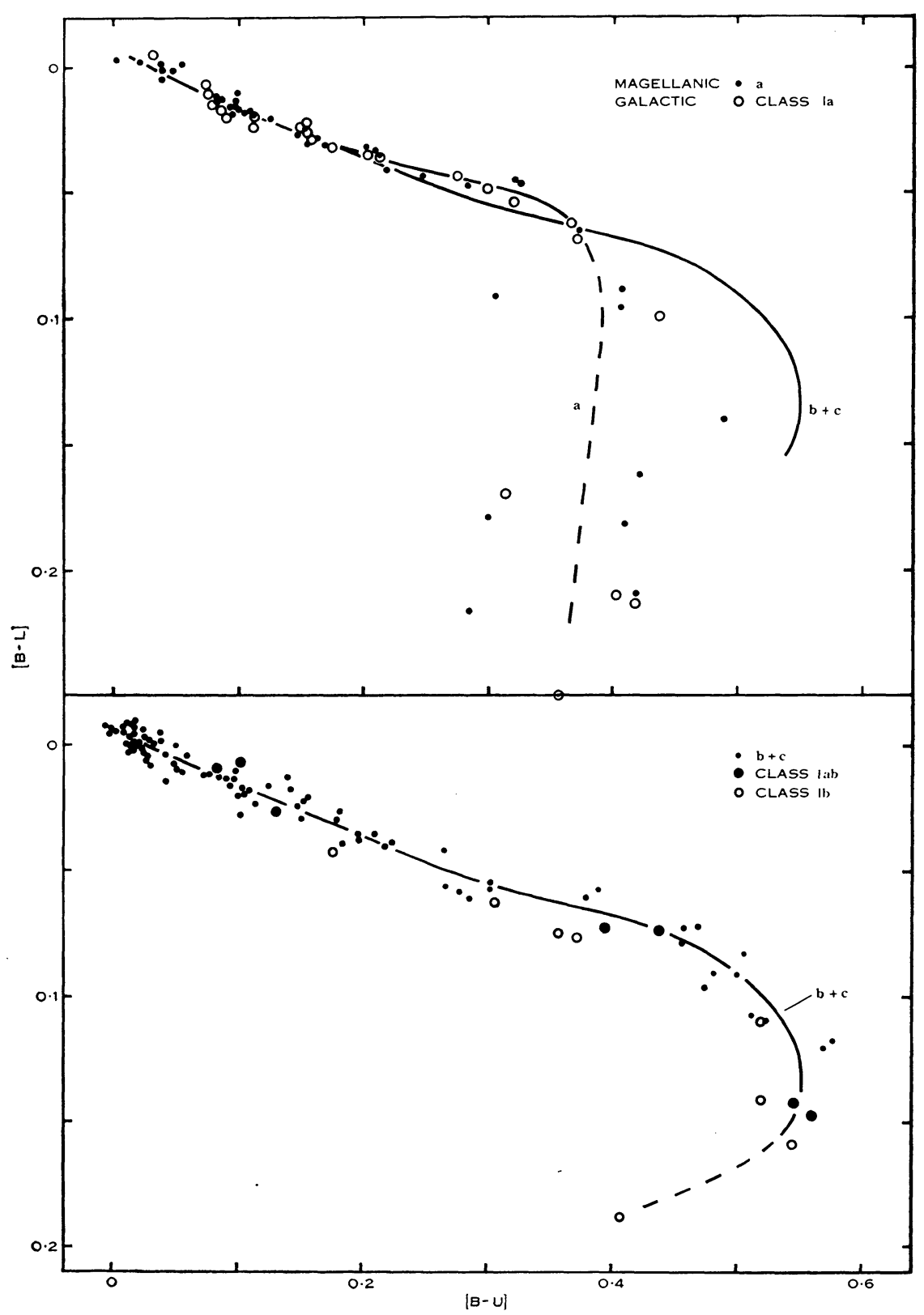

Fig. 3.-Reduced colour-colour diagram $[B-L]-[B-U]$. Lines are mean lines $a$ and $b+c$ for Magellanic stars. 
Galactic supergiants with known spectral type are also shown in Figure 4. The stars of luminosity class Ia are shown as filled dots, class Iab as half-filled dots, and class $\mathrm{Ib}$ as open dots.

Spectral types are represented by the short crossing lines. Only A0 stars are sufficiently numerous to show the slope and curvature of the lines. For the other classes the lines are uncertain and are arbitrarily drawn more or less parallel

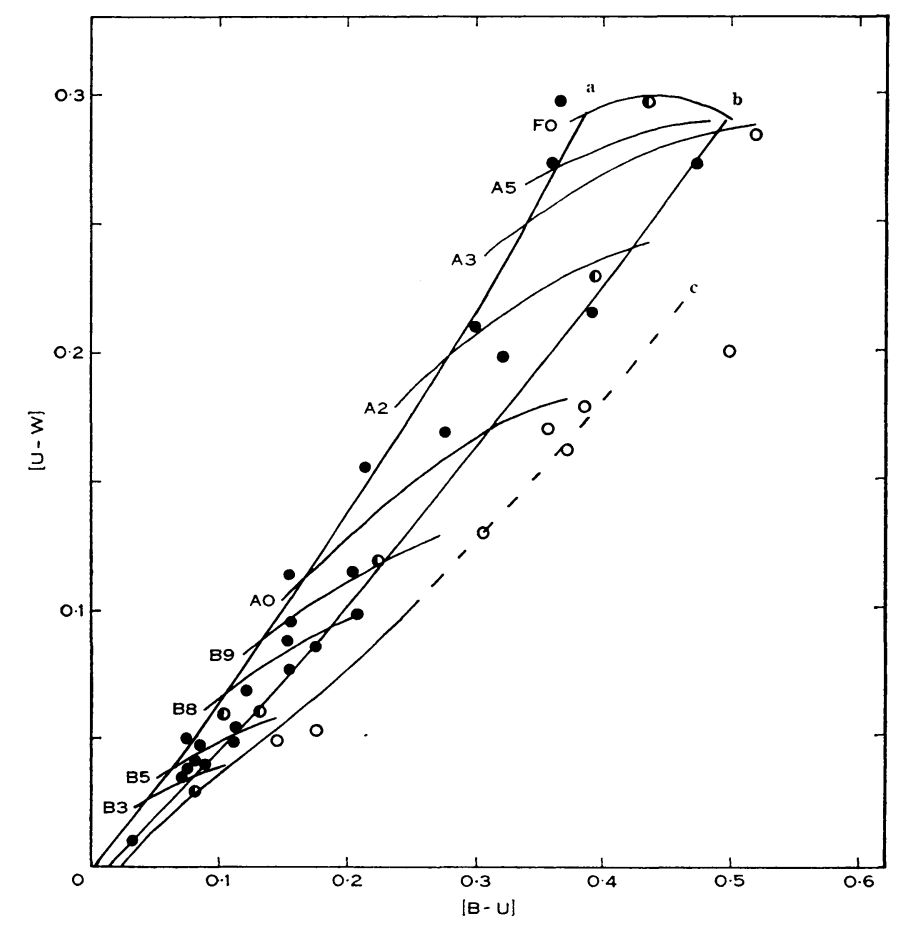

Fig. 4.-Reduced colour-colour diagram $[U-W]-[B-U]$.

to each other. The deviations of the observed spectral types both for the Clouds (Feast, Thackeray, and Wesselink 1960) and for the galactic stars are on the average one-tenth of a spectral class.

\section{Conclusion}

It has been shown that in all possible ways where a comparison can be made, the colours of the galactic supergiants are identical with those of the Magellanic Cloud supergiants. This suggests that there is no essential difference in the structure or chemical composition. It might be possible that in the colours the effects of different composition are too small to be observed. However, the spectral types are classified with the strength of the metallic lines as a criterion, and in the relation between colour and spectral type the galactic and Magellanic stars are also alike. This makes a difference in chemical composition unlikely. Finally the colour-magnitude diagram shows, at least for the Magellanic field stars which we observed, with its absence of yellow giants, a distribution with a wide Hertzsprung gap which strongly 
resembles that of the Galaxy. The conclusion of this study therefore is that no real difference can be detected between the Galaxy and the Magellanic Clouds. The only notable difference observed is the apparent lack of interstellar dust in the Clouds outside the 30 Doradus region. However, since dense concentrations of stars were avoided in the observations, this conclusion is not definite.

\section{References}

Feast, M. W., Thackeray, A. D., and Wesselink, A. J. (1960).-M.N. 121 : 337-85.

DE JAGER, C., and Neven, L. (1957).--Recherches Astron. Obs. Utrecht 13, No. 4.

Johrson, H. L. (1958).-Lowell Obs. Bull. 4: 37-46.

Walraven, Th., and Walraven, J. H. (1960).-B.A.N. 15: 67-83.

\section{Discussion}

Eggen: It appears that your separation between luminosity classes in the early-type supergiants is larger than previously supposed so that the absorption for galactic supergiants has been overestimated. If so, the luminosity of the galactic supergiants has been overestimated.

Walraven: I used only the galactic absorption which affects the Clouds; if this quantity is reduced, the distance modulus of the Clouds becomes larger.

I have not determined the absorption of galactic supergiants.

Feast: In view of the fact that the fainter stars studied by Dr. Walraven are concentrated in an area far out from the bars of the LMC and SMC where absorption is probably extremely small whilst the brighter stars are distributed over the whole area of both Clouds, it seems possible that some of the difference between the faint and bright stars reported by Dr. Walraven may be due to the effects of interstellar absorption.

Walraven : If absorption is present it is difficult to decide how much, because we have no basis for comparison. We concluded that the absorption is low from the very small scatter in colour. All the points fall very close to the mean line, which shows a scatter not larger than the uncertainty in luminosity. No residual to be accounted for by absorption remains. Only stars near 30 Doradus show absorption.

$A r p:$ In the Johnson and Morgan $U-B, B-V$ system the $\mathrm{F}$ supergiants have increasing amounts of ultraviolet deficiency (measured from the luminosity class $\mathrm{V}$ stars). This $U-B$ deficiency increases in amount as the luminosity class goes brighter as would be expected from the decreasing electron pressure. Dr. Walraven's results go in the opposite sense which seems puzzling. Could this be a reddening effect?

Walraven: According to theoretical models the ultraviolet deficiency indeed increases steadily with decreasing gravity. It is remarkable that our observations go in the opposite sense. It could be reddening, it could be hydrogen emission, but in both cases it should be then an intrinsic property of the stars.

It is also possible that the range of computed models does not extend far enough to extremely low values of gravity.

Arp : I would expect them to fall even further below.

Walraven: The observations show very convincingly that a maximum Balmer jump is reached at luminosity class Ib.

Blaauw: There seems to be a lower envelope of the points in the colour-magnitude diagram of the SMC. Do you think this is real, or might it be due to the way these stars were selected?

Walraven: It need not be real as the faint stars are observed mainly in associations, while the bright supergiants are more scattered over the area of the Clouds.

Blaauw: In your plot of the quantity in brackets is this free of reddening?

Walraven: We assumed the slope of the reddening line from $\mathrm{O}$ stars in the Galaxy. 
Blaauw: Would a difference between the reddening laws of the Magellanic Clouds and the Galaxy affect the results for your quantities in brackets?

Walraven: The reddening in the Clouds is so small that it does not matter.

$B o k$ : All of us who work in the standard $U B V$ colour system with its problems in the ultraviolet will wish to congratulate Dr. and Mrs. Walraven on their fine photometric work. The three colour ranges $U, W$, and $L$ will surely play important roles in the future.

Graham: I would like to comment briefly on the remarks made by Dr. Walraven about the Stark effect in the atmosphere of supergiants. I have not observed as many supergiants as Dr. Walraven but $I$ have not found any evidence for the $\mathrm{H} \beta$ index ceasing to be a good luminosity parameter except in the very brightest supergiants when emission effects become prominent. On using the $\mathrm{H} \beta$ calibration procedure described last week, a mean uncorrected distance modulus of $18 \mathrm{~m}^{4}$ with a mean internal error of $0 \mathrm{~m}_{4}$ was derived for a few supergiants in the wing of the Small Cloud. Because of the several assumptions involved in the use of such a calibration in the Clouds, I do not think this modulus should be taken any more seriously than as indicating an apparent distance modulus of less than $19^{\mathrm{m}} .0$.

Walraven: The accuracy of the $[B-L]-[B-U]$ lines is very high, the mean deviation is only 0 m 005 , which is much better than for the other colour relations. This also suggests that there is generally a close relation between the strength of the higher Balmer lines, observed in $L$, and the strength of the Balmer jump, observed in $U$. It is not impossible however that for $\mathrm{H} \beta$ which Mr. Graham used, the situation is different from that of the higher Balmer lines.

Eggen: It is worth noting that the $\mathrm{H} / \mathrm{He}$ ratio can also cause a variation in the $(U-B)$, $(B-V)$ diagram.

Aller: We have no sound basis for theoretical calculations of model atmospheres for supergiants. The complexities shown in the K-type supergiants such as 31 Cygni, i.e. detached prominences and filaments of large optical depth, suggest that the usual assumption of hydrostatic equilibrium is not valid. One could perhaps employ crude, approximate models, but it is hard to know how far to trust them.

Pagel: While agreeing completely with Dr. Aller's remarks as to the difficulty in constructing a logical model, it is very tempting to examine the possibility of explaining some of the effects that we have heard about by rough arguments. Both the reduction in the Balmer jump and the extreme increase in redness needed to go from type $F$ to $G$ seem to be compatible with electron scattering opacity.

Walraven: Indeed the colours suggest that the brighter stars have electron scattering since the spectral features disappear.

Westerlund: We all agree that the most luminous supergiants are difficult to use for distance determinations. What distance moduli for the LMC and SMC do you derive using the fainter stars $(m \sim 14-15)$ in your diagrams?

Walraven: We have not used the faint blue stars. For such faint stars the accuracy of the observations is low and the luminosity effects at B0 are small. However, if we assume them to be of class $\mathrm{V}$, their brightness has approximately the right value.

Tiff: : The moduli of about 18.5 being discussed for the Clouds from supergiants is, as one might expect, in agreement with the cepheids. It is not necessarily in agreement with other types of stars.

de Vaucouleurs: In 1959 a quantitative analysis of the equivalent widths of sensitive lines, including HeI 3820, in the spectrum of the bar of the Large Cloud, showed that a good fit could be obtained by a suitable mixture of galactic stars of various spectral types (especially $B$ and $K$ ). The close agreement suggests that no large differences in $\mathrm{He}$ and metal abundances with respect to $H$ can exist between galactic stars and Cloud stars.

In 1955 an apparent photographic modulus of $18 \cdot 7 \pm 0 \cdot 2$ p.e. was derived by considering all available criteria (cepheids, RR Lyrae, novae, globular clusters, HII regions, etc.). This is better than placing all one's faith in only one criterion or another. All recent evidence confirms this value. It seems difficult at present to justify a distance greater than $50 \mathrm{kpc}$.

Walraven: Then the Johnson and Iriarte luminosity classification is about right. 
Tifft: If $18 \cdot 5$ is taken as the Cloud modulus the cluster type variables may become uncomfort. ably faint $\approx+0 \cdot 8=M_{V}$.

Blaauw: I doubt whether the available evidence on the absolute magnitude of the RR Lyrae in particular is strong enough to cause any concern about the different distance moduli derived from these objects in the Clouds and the supergiants.

Walraven: The galactic supergiants may be underestimated.

Arp: Determinations from globular clusters give $M_{V}=+0^{\mathrm{m}} \cdot 3$ and $M_{B}=+0^{\mathrm{m}} 5$ as the best values for the $R R$ Lyrae stars. This gives a reasonable value for the galactic centre distance fainter values for the RR Lyrae stars would give an even smaller distance than $8 \cdot 2 \mathrm{kpc}$.

Blaauw: Considering the probable errors there is no basis for concern.

Arp : A point of more uncertainty is that there are only a few RR Lyrae stars in the Clouds and they may be unrepresentative.

\title{
67. SPECTROSCOPIC WORK IN THE MAGELLANIC CLOUDS: NGC 330 IN THE SMC
}

\author{
M. W. Feast \\ Radcliffe Observatory
}

This paper gives the results of a spectroscopic study of individual stars in the SMC cluster NGC 330. Before this, however, it may be useful to review briefly the spectroscopic evidence bearing on the questions of reddening and chemical composition in the Magellanic Clouds-matters about which there has not been entire agreement in the past.

Earlier Radcliffe results (Feast, Thackeray, and Wesselink 1960) showed that for the early-type supergiants in any spectral class the least reddened stars in the Magellanic Clouds agreed well in colour with Johnson's (1958) intrinsic colours except that amongst the A type supergiants Feinstein's (1959) intrinsic colours were to be preferred. The Radcliffe results gave a clear indication of interstellar reddening in both Clouds, although the mean total absorption was small for the stars studied ( $\sim \frac{1}{3}$ magnitude). Various lines of evidence lead to the conclusion that these reddenings are real and not, for instance, due to errors in the spectral types or intrinsic colours.

(1) Reddening is found from $U B V$ photometry as well as from spectral types and $(B-V)$ colours, as Wesselink's (1962) recent work amply confirms.

(2) The possibility that the wide range in $(B-V)$ observed at a given spectral type amongst the Radcliffe stars might be due to a strong dependence of intrinsic colour on absolute magnitude, with essentially no reddening, has been investigated. However, the correlation which would then be expected within a given type between $(B-V)$ and magnitude is not found. An apparent exception to this result occurs amongst the LMC B0 stars. In this spectral class three stars with $V$ near 12.55 are systematically bluer than three with $V$ near $11 \mathrm{~m} 5$. However, the three faint stars are all in the region near HDE 271191, which is on the outskirts of the LMC where the mean absorption is known to be low.

(3) The LMC star with the greatest known reddening was studied chiefly because it lay in an apparent absorption lane. Furthermore, the reddening is sys- 